\title{
SALUD MENTAL Y NUEVAS COMPLEMENTARIEDADES TERAPÉUTICAS. LA EXPERIENCIA EN DOS HOSPITALES PÚBLICOS DE BUENOS AIRES, ARGENTINA
}

SAÚDE MENTAL E NOVAS COMPLEMENTARIDADES TERAPÊUTICAS. EXPERIÊNCIA EM DOIS HOSPITAIS PÚBLICOS DE BUENOS AIRES, ARGENTINA

\author{
MENTAL HEALTH AND NEW THERAPEUTIC COMPLEMENTARITIES. THE
} EXPERIENCE IN TWO PUBLIC HOSPITALS IN BUENOS AIRES, ARGENTINA

María Mercedes Saizar

Consejo Nacional de Investigaciones Científicas y Técnicas y Centro Argentino de Etnología Americana,

Buenos Aires, Argentina

\author{
Mercedes Sarudiansky y Guido Pablo Korman \\ Consejo Nacional de Investigaciones Cientificas y Técnicas y Universidad de Buenos Aires,
}

Buenos Aires, Argentina

\section{RESUMEN}

En las últimas décadas las terapias alternativas se han convertido en una opción cada vez más frecuente entre la población general de las ciudades industrializadas, aunque su aceptación como opciones válidas en el sistema de salud aún se encuentra en discusión. A pesar de ello, en la Ciudad de Buenos Aires (Argentina) han surgido talleres dentro de los hospitales generales que ofertan terapias alternativas/complementarias, asociadas a servicios o profesionales de la salud mental. A partir de un estudio cualitativo en dos hospitales públicos de la Ciudad Autónoma de Buenos Aires, indagaremos los motivos que dan origen a dicho fenómeno, destacando las formas de expresión que adquiere y la complejidad de un campo en construcción.

Palabras clave: terapias alternativas; salud mental; hospitales públicos; Buenos Aires; Argentina

\section{RESUMO}

Nas últimas décadas, terapias alternativas têm se tornado cada vez mais comuns entre a população geral em cidades industrializadas, embora sua aceitação como opções válidas no sistema de saúde ainda esteja em discussão. No entanto, na Cidade de Buenos Aires (Argentina), oficinas emergiram dentro de hospitais gerais que oferecem terapias alternativas / complementares, serviços associados e profissionais de saúde mental. A partir de um estudo qualitativo em dois hospitais públicos da Cidade Autônoma de Buenos Aires, vamos investigar as razões que dão origem a este fenômeno, destacando as formas de expressão que adquirem e a complexidade de um campo em construção.

Palavras-chave: terapias alternativas; saúde mental; hospitais públicos; Buenos Aires; Argentina.

\begin{abstract}
In recent decades, alternative therapies have become an increasingly common option among the general population in industrialized cities, although their acceptance as valid options in the health system is still under discussion. Nevertheless, in the City of Buenos Aires (Argentina) workshops have emerged within general hospitals that offer alternative / complementary therapies, associated to mental health professionals and departments. From a qualitative study in two public hospitals in the Autonomous City of Buenos Aires, we will investigate the reasons that lead to this phenomenon, emphasizing the forms of expression they acquire and complexity of a field under construction.
\end{abstract}

Keywords: alternative therapies; mental health; public hospitals; Buenos Aires; Argentina 
Las terapias alternativas/complementarias suelen considerarse como un conjunto amplio de recursos curativos que presenta teorías, prácticas y modalidades de atención diferentes de aquellas oficialmente reconocidas, a pesar de que sus límites tanto internos como respecto de las demás medicinas son difusos y posiblemente cambiantes (Zollman \& Vickers, 1999). La expansión en lo que hace a la utilización de estas terapias en distintas sociedades ha merecido la atención de investigadores de disciplinas del campo de las ciencias sociales -especialmente de la sociología y de la antropología médica- y de profesionales e investigadores del área de la salud. En diferentes investigaciones se manifiesta claramente su creciente utilización, tanto en estrategias de complementariedad terapéutica con la biomedicina como en estrategias de uso paralelo y exclusivo. Los motivos de selección de las terapias no convencionales han sido atribuidos a diversos factores, entre los que se destacan la insatisfacción con el modelo biomédico de atención de la salud-enfermedad (Boon, Brown, Gavin, Kennard, \& Stewart, 1999), la demanda de un nuevo tipo de relación con el especialista terapéutico y a los límites del paternalismo biomédico (Montgomery, 2006), la aparición de nuevas subjetividades que, en el contexto de la posmodernidad, asignan un rol central de responsabilidad al sujeto en el cuidado de su salud (Siahpush, 1998), la búsqueda de nuevos estilos de terapia más suaves, holísticos y menos invasivos que la biomedicina alopática (Douglas, 1998; Vincent \& Furnhman, 1996), el surgimiento de movimientos sociales asociados a la Nueva Era (Albanese, 1990; Carozzi, 2001; Shimazono, 1999), entre otros. Ha sido señalado también el importante rol que, desde la perspectiva de los usuarios, cumplen estas medicinas frente al tratamiento de enfermedades graves $\mathrm{o}$ crónicas, tales como el caso del cáncer (Broom \& Tovey, 2008), el asma (Freidin \& Timmermans, 2008), cardiopatías (Ai \& Bolling, 2002), sintomatología "ambigua" (Karasz \& Dempsey, 2008), hasta distintos trastornos mentales, como depresión (Berenzon Gorn, Navarro, \& Saavedra Solano, 2009) y trastornos de ansiedad (Eisenberg et al., 1998). De hecho, de acuerdo a varios estudios internacionales, la mayoría de los pacientes con trastornos mentales acuden a una utilización mayor de tratamientos alternativos (p.e. Jorm, 2000; Mamtani \& Cimino, 2002) o a alguna medicina no convencional. En un estudio realizado en Gran Bretaña, Cartwright y Torr (2005) señalaron que estas terapias no sólo cumplen una importante función en tanto ayudan a afrontar el estrés que acompaña los tratamientos de largo plazo o de características invasivas, sino que fundamentalmente otorgan a los dolientes la experiencia de involucrarse más activamente en el proceso de tratamiento de la enfermedad.

Una cuestión interesante descansa en el hecho de que, mientras que la utilización de las terapias alternativas/complementarias es un hecho ampliamente demostrado y consensuado por la comunidad de cientistas sociales, la definición acerca de cuáles son y bajo qué categoría agruparlas continúa siendo un motivo de debate; debate que, al interior de este ámbito, aún no ha sido resuelto (Broom \& Tovey, 2008).

Una de las definiciones más utilizadas y que se puede hallar en numerosos trabajos de investigación es la de la Organización Mundial de la Salud (2002), que propone denominarlas medicinas alternativas/ complementarias -MAC, por sus siglas en español-, en virtud de su utilización respecto de la biomedicina. En Estados Unidos, el Centro Nacional de Medicina Complementaria y Alternativa-NCCAM- ha definido a la medicina alternativa "como un grupo de sistemas, prácticas y productos médicos y del cuidado de la salud que no son actualmente consideradas como parte de la medicina convencional" (National Institute of Health, 2003). Algunos autores han problematizado esta categoría al señalar que la utilización de las terapias en las trayectorias individuales es lo que permite designarlas como complementarias o alternativas respecto del tratamiento biomédico (Broom \& Tovey, 2008), mostrando los límites de las clasificaciones de tipo etic y señalando la importancia de rescatar la experiencia de los actores sociales al momento de considerar tales distinciones.

Otras líneas de análisis han denominado "medicinas alternativas" a aquellas que se presentan como un recurso paralelo de atención de la salud para sectores urbanos medios y medio altos, que manifiestan además una alternancia convergente entre la atención biomédica arancelada y los recursos de origen exótico (Alexander, 1992; Heelas, 1996; Martins, 1999; Shimazono, 1999). Al respecto, se ha señalado que dada la creciente expansión del consumo de este tipo de prácticas terapéuticas constatada en la última década y en virtud de su inclusión en variados sistemas de salud pública - como son el caso de Canadá, Estados Unidos, Alemania, Australia, Reino Unido, Brasil e Israel-, la preferencia por las terapias alternativas no sería exclusiva de determinados sectores sociales (Mc Guire \& Kantor, 1988; O'Connor, 1995) sino que se asociaría más bien a determinados estilos culturales (Douglas, 1998) y a nuevos modos de comprender la salud, la enfermedad y el proceso terapéutico (Furnham \& Smith, 1988). Las terapias alternativas, asimismo, conviven en este complejo campo con otras ofertas 
terapéuticas (tales como la biomedicina, las medicinas tradicionales, las medicinas religiosas y los diversos autotratamientos), participando de una configuración etnomédica (Idoyaga Molina, 2008) en el que se inscribe el fenómeno de la complementariedad, tema que desarrollaremos a continuación.

\section{Combinación de medicinas}

La complementariedad terapéutica es un fenómeno ampliamente documentado en distintas sociedades. Implica la combinación, frente a un mismo episodio de enfermedad, de estrategias terapéuticas diversas (Fadlon, 2005; Sharma, 2000). En la Argentina distintos investigadores han señalado la existencia de la complementariedad terapéutica entre individuos de distintos sectores sociales y pertenecientes a sociedades tanto urbanas como rurales (Korman \& Idoyaga Molina, 2010; Saizar, 2009; Freidin \& Timmermans, 2008), entre los que se han distinguido variadas estrategias de selección, combinación y rechazo de las diversas ofertas presentes. Las mismas han sido explicadas por la incidencia de factores culturales, económicos, políticos, étnicos, religiosos y estilos de pensar (Idoyaga Molina, 1997). Respecto de la complementariedad terapéutica, la norma general en todos los casos estudiados pareciera indicar un uso pluralista más que una excepción.

A pesar de la existencia de este fenómeno y de las recomendaciones que la Organización Mundial de la Salud realizara en el año 2002 respecto de propiciar el respeto por las diversas formas culturales de la medicina, en la Argentina -un país con marcada diversidad cultural- la biomedicina es la única opción en salud ofertada por el Estado y oficialmente legislada. El hospital público, ámbito en el que el Estado oferta atención de la salud de manera gratuita para todos los habitantes- se constituye así en el lugar por excelencia donde la lógica biomédica actúa, regulando la oferta de prácticas médicas y siendo el único "arte de curar" habilitado para intervenir sobre los cuerpos de los enfermos, según se enmarca en la Ley Nacional de Ejercicio de la medicina, odontología y actividades auxiliares ( $\mathrm{N}^{\circ}$ 17.132) sancionada en el año 1967. Sin embargo, en los últimos años algunos hospitales de la Ciudad Autónoma de Buenos Aires han desarrollado y puesto en funcionamiento una oferta de talleres de terapias alternativas, que se ofrecen de forma gratuita a la población asistente. La distancia entre lo escrito en la ley que regula la práctica médica y la oferta real nos lleva a preguntarnos ¿Cómo es que conviven estas miradas distintas en el espacio de lo público? ¿En qué marco institucional se inscriben?
Intentando responder a estos cuestionamientos, en esta oportunidad nos proponemos indagar la utilización de terapias alternativas en ámbitos hospitalarios. Indagaremos respecto de los motivos que habilitaron la ejecución de talleres de terapias alternativas en el contexto hospitalario, la aceptación por parte de los terapeutas alternativos de dictar los mismos en el ámbito biomédico y los motivos de participación de los usuarios.

\section{Hospitales y talleres en Buenos Aires: reflexión, comunidad y alternativas}

Los talleres comunitarios que funcionan en los hospitales públicos de Buenos Aires se integran principalmente con el área de Salud Mental de cada institución y se promueven a través del Gobierno de la Ciudad de Buenos Aires como Talleres de Promoción en Salud Mental. Estos talleres se promocionan como espacios grupales de promoción de la salud mental, cuyo objetivo es generar en sus participantes un estado anímico favorable para atravesar las situación que viven.

Si bien la oferta de talleres se presentan como una serie homogénea de prestaciones gratuitas dentro de esa oferta, se distinguen tres tipos de grupos: Un primer grupo que se enmarca en el tratamiento de distintas experiencias conflictivas de la vida de los participantes, que exceden el marco de lo meramente biológico e incorporan aspectos relativos a las emociones, los vínculos y el desarrollo de habilidades sociales. Algunos de ellos son: Relación con los hijos adultos, Vínculos con el sexo opuesto, Duelo por la muerte de seres amados, Pareja y Vida Cotidiana, entre otros.

Un segundo grupo, conformado por talleres que ofrecen espacios de reflexión y contención del paciente y su familia, frente a las situaciones vitales críticas que devienen del padecimiento de enfermedades definidas por la biomedicina. Ejemplo de ello son los talleres: Viviendo con VIH-Sida, Grupos para pacientes Diabéticos, Obesos Recuperados, entre otros. En todos ellos el objetivo es brindar contención emocional y psico-educación del paciente y su familia, mediante la aplicación de herramientas para afrontar su enfermedad y lograr así una mayor calidad de vida.

En términos de Illouz (2010), ambos grupos manifiestan una conformación heterogénea -de género, edad, instrucción formal- pero comparten una misma narrativa temática, que puede ser la obesidad, la pérdida de seres queridos, etc. Comparten, asimismo, la condición de estructurar o delimitar las narrativas de 
los participantes mediante un eje discursivo que define lo normal y lo patológico desde un sentido biomédico (Saizar, Bordes, \& Sarudiansky, 2010). Si bien estos grupos suelen estar coordinados por profesionales biomédicos del área de salud mental, se definen como espacios de contención, fortalecimiento del lazo social y búsqueda de soluciones a determinadas problemáticas, rechazando su encuadramiento en las definiciones de "talleres asistenciales o psicoterapias grupales".

El tercer grupo se compone de talleres que brindan una oferta de terapias alternativas/complementarias, ajenas al campo biomédico local. En este contexto, se ofrecen diversos talleres, tales como Shiatsu, Cuencos Tibetanos, Biodanza, Danzas Circulares, Yoga, Reflexología Holística, Masajes Shantala, Tai Chi Chuan, Meditación con Mandalas, Bioenergética, Astrología, Digitopuntura, etc.

\section{Metodología}

La metodología que se utilizó para llevar adelante este trabajo parte de un enfoque fenomenológico y cualitativo, con el objetivo de comprender los significados asociados a la salud y la enfermedad por parte de efectores y usuarios de prácticas alternativas en los hospitales públicos de la Ciudad Autónoma de Buenos Aires. Esta metodología utiliza un razonamiento inductivo, que prioriza los datos recolectados en el campo para generar hipótesis. La investigación parte de la posición de que una comprensión interpretativa es solamente posible a partir de deconstruir los significados del fenómeno (Thorne, 2000).

Para llevar adelante este estudio, seleccionamos dos hospitales de agudos que ofertan talleres de terapias alternativas al público en general de la Ciudad Autónoma de Buenos Aires. La suma de pacientes aproximada por año de ambos hospitales es de 120.000 pacientes. Fueron entrevistados los dos coordinadores generales (en un caso una psiquiatra y en el otro una psicóloga), quienes funcionan como nexo entre los distintos servicios de cada hospital -en especial el Servicio de Salud Mental- y los Talleres Alternativos. Entrevistamos también a ocho efectores de talleres -cuatro de cada hospital- entre los que encontramos especialistas alternativos de las siguientes terapias: danzas circulares, cuencos tibetanos, yoga, tai-chichuan, meditación trascendental, bioenergética y reflexología.

El número de asistentes a los talleres de los hospitales seleccionados es de aproximadamente 270 personas. El grupo de usuarios pertenece a los denominados sectores medios y medios bajos, con niveles de instrucción que varían entre secundaria incompleta y universitaria de grado. Las edades varían en un rango de entre 42 y 81 años, con mayoría de mujeres mayores de 65 años. Es interesante señalar que de los 270 participantes, sólo 32 eran varones y que todos ellos eran mayores de 65 años. Si bien dialogamos acerca de los temas que son de interés con la mayoría de los usuarios, seleccionamos a diez asistentes a los talleres - cinco de cada hospital- con el objetivo de ser entrevistados en profundidad. La selección se realizó en virtud de su participación activa en la oferta de talleres -lo cual era observado también a partir de la asistencia ininterrumpida a cada taller- y la disponibilidad y predisposición para las entrevistas, que se realizaron fuera del ámbito hospitalario.

Las entrevistas a coordinadores, especialistas alternativos y usuarios fueron semi-estructuradas sobre la base de una serie de preguntas abiertas acerca de la utilización de estas estrategias en el contexto hospitalario. Se consideraron las siguientes temáticas en relación con los coordinadores y los especialistas alternativos: (a) el origen de los talleres, (b) la organización de los talleres, y (c) motivos de recomendación a los talleres. En las entrevistas con los usuarios se consideraron los siguientes aspectos: (a) Las razones de participación y asistencia; (b) las definiciones de salud y enfermedad y (c) las reflexiones respecto de la experiencia de combinar tratamientos. El trabajo de campo se desarrolló en el período comprendido entre abril del 2009 y agosto del 2010. Los materiales fueron audiograbados y transcriptos literalmente. La duración de las entrevistas varió entre 30 y 90 minutos. Previo a los encuentros, tanto de las entrevistas como de la observación participante en los talleres, se contó con la autorización y consentimiento informado de todos los participantes. Los nombres de los informantes fueron cambiados para preservar su identidad.

\section{Resultados}

A partir de los datos relevados en nuestro trabajo de campo, expondremos los resultados de nuestro análisis siguiendo el ordenamiento propuesto por los ejes que estructuraron las entrevistas y las observaciones realizadas. Ejemplificaremos, asimismo, con relatos de los distintos entrevistados -coordinadores, talleristas y participantes.

\section{Coordinadores y talleristas}

a) Origen de los talleres

En las entrevistas con los coordinadores pudimos detectar dos grandes ideas que explican el origen 
de los talleres. La primera mención es que permiten la creación de un ámbito que acompaña la oferta del área de Salud Mental, brindando a los pacientes actividades que les permiten participar de un grupo, establecer vínculos sociales y crear hábitos saludables, funcionando como una estrategia complementaria de las prácticas de Salud Mental. Este aspecto coincide, en cierta medida, con el objetivo explicitado en el portal web del Gobierno de la Ciudad, mencionado anteriormente.

"La idea que nos condujo a crear estos espacios fue la de brindar talleres que permiten trabajar la grupalidad, como complemento de los tratamiento psicológicos y psiquiátricos, pero desde una óptica más amplia" (María, coordinadora).

se despierta la posibilidad de comunicación, la gente empieza a sentir que no está sola, empieza a darse cuenta que su problema no es tan grave como creía, la gente se empieza a dar cuenta que al otro le pasan cosas parecidas. Además, el grupo funciona como un espejo... para ver que uno no está solo. (Julieta, coordinadora).

Paralelamente, también se explicita la promoción de la búsqueda de bienestar y de la salud en general por sobre el tratamiento específico de la enfermedad.

Estos espacios permiten pensar al hospital desde un lugar distinto, ya no se lo asocia al estar enfermo, sino a tratar de sentirse mejor, de buscar calidad de vida... cambió la mirada que la gente tiene del hospital como lugar asociado a la muerte y hay una política del gobierno que promueve estas acciones, sin embargo antes de que los políticos lo hubieran pensando nosotros lo estábamos haciendo espontáneamente. (Julieta, coordinadora)

Desde la perspectiva de nuestros entrevistados, los talleres comenzaron a funcionar con anterioridad a la creación de la política de salud tendiente a promocionar el bienestar. Fueron diseñados y puestos en marcha por distintos profesionales de la salud -en especial, de la salud mental- como una forma de promover la grupalidad, enfatizando el aspecto terapéutico asociado al hecho grupal como el fundamento de la eficacia de esos espacios. Es en una segunda instancia que se enmarca la actividad en cuestión en el contexto de una decisión política de promoción de la "salud anímica" de la población., cuestión que coincide con una tendencia a la psicologización de las actividades alternativas por parte de los coordinadores, quienes justifican su utilidad y aceptación a partir de nociones del campo psicológico-psiquiátrico (Korman \& Saizar, 2006).

Sin embargo, estas ideas sobre el origen de los talleres no es del todo compartida por todos los participantes de los mismos. Los especialistas alternativos que dictan los talleres plantean una perspectiva diferente:

María tiene una mirada muy abierta respecto de lo que es la salud. Cuando nos convocó, nos explicó que le interesaba ofrecer un espacio diferente para los pacientes, donde pudieran trabajar la salud desde una mirada holística, más en consonancia con la idea de que la salud es más que no estar enfermo. (Alejandra, tallerista de Danzas Circulares)

Julieta siempre nos dice que el hospital tiene que
cambiar, tiene que ocuparse del bienestar de los
vecinos y no sólo de tratar enfermedades... Ella
cuando nos juntamos los sábados con todos los
talleristas hace mucho hincapié en que no hagamos
psicoterapia, que promocionemos conductas más
saludables y que usemos un lenguaje sencillo y
claro, que evitemos términos técnicos de nuestras
especialidades. (Roberto, tallerista de Meditación
Trascendental)

En los relatos podemos ver cómo se atribuye la creación de estos espacios al interés particular de los coordinadores, como poseedores de una mirada holística respecto de la salud y la enfermedad, que permite ampliar la perspectiva tradicional hospitalaria.

Entonces, existe un contrapunto entre la perspectiva de los coordinadores y la de quienes dictan los talleres alternativos. En los primeros se advierte una lógica de domesticación, en términos de Fadlon (2005), de las terapias alternativas, las que serían aceptadas en tanto sus efectos pueden comprenderse como coadyuvantes del tratamiento biomédico, despojándose de aspectos esotéricos y resaltándose sus aspectos más tangibles. En tanto, los especialistas alternativos lo perciben como una posibilidad de legitimar estas prácticas, que les permite negociar con el campo biomédico los alcances y límites de su incorporación al sistema hospitalario (Broom \& Tovey, 2008).

\section{b) Organización de los talleres}

En ambos hospitales, la organización y la coordinación de los talleres depende de un profesional de la Salud Mental, cuyas tareas incluyen la selección de los especialistas alternativos, la diagramación de las actividades anuales y la acreditación de los dictantes de los talleres.

Cada año, a principio de año, organizamos la oferta de actividades. Convocamos a gente interesada en dictar talleres. Les pedimos que nos traigan un curriculum $y$ después los entrevistamos. Es muy importante para nosotros que acrediten una formación en la práctica que van a desarrollar, que hayan estudiado y que tengan acreditación para eso. Queremos gente 
seria, que pueda desempeñarse con responsabilidad. (Julieta, coordinadora)

Yo llamo gente que me recomendaron los que ya están trabajando, que sé que trabajan bien y que son serios en lo que hacen. Los entrevisto, les pido que me traigan sus certificados de estudio $y$ después, informo a la Dirección los nombres de los seleccionados. Para mí es muy importante contar con un buen equipo de talleristas, calificados en su arte. (María, coordinadora)

El proceso que realizan los coordinadores respecto de los antecedentes de los postulantes supone un método de evaluación y la presunción de que un especialista de la biomedicina estaría avalado para acreditar la idoneidad de un especialista de una disciplina alternativa. Este régimen implica un marco de evaluación y acreditación que los coordinadores deben observar para conservar el encuadramiento de su trabajo.

\section{c) Motivos de recomendación}

Los coordinadores coinciden en que el principal motivo de derivación de pacientes a los grupos alternativos es ofertarles una actividad complementaria al tratamiento -principalmente psicológico o psiquiátrico- y que valoran como positiva desde diversos aspectos: posibilitarle un espacio de sociabilidad, compartir con otros pares circunstancias y experiencias vitales similares, beneficiarse de técnicas de relajación y realizar ejercicios físicos suaves.

Al psicótico, lo mandás al taller donde no hay que hablar, como el de Tai Chi Chuan o Yoga y ya está. Al depresivo, no lo dejes hablar ni por broma, porque te va a inundar de nostalgia y de angustia y todos van a llorar y no es el lugar. Le recomendás actividad física, como puede ser Yoga o bioenergética. (Julieta, coordinadora)

Puede observarse, entonces, que las ventajas se asocian con la combinación del tratamiento psicoterapéutico o psiquiátrico con determinadas disciplinas que poseen en común el proveer de técnicas que hacen al uso del espacio grupal y la actividad física. Por otra parte, los coordinadores explican que, como efecto secundario, los talleres permitieron dar respuesta a la demanda de atención, pero sobre todo de contención emocional que exigen los pacientes crónicos:

Esto empezó cuando empecé a ver que los pacientes crónicos, los psicóticos, venían, empezaban a dar vueltas alrededor del servicio. Un día venían para pedir una receta, otro día venían porque se olvidaron de algo, otro día venían por otra cosa. Entonces, personalmente, me di cuenta de que el paciente venía porque el hospital era un lugar de referencia, un lugar para estar, fundamentalmente pacientes crónicos que no tienen un lugar en el mundo, porque la cronicidad hace que se pierdan los lugares de referencia laborales, académicos, la familia los tiene apartados. (Julieta, coordinadora)

El paciente crónico se representa como aquel que debería concurrir una o dos veces por semana a la consulta con el psiquiatra y/o el psicólogo y que, pese a esta disposición, asiste habitualmente, generando la saturación del servicio, debido a que permanece deambulando por la institución. Los coordinadores incluyen a estos usuarios como asistentes de los talleres, aprovechando las facilidades que brinda su permanencia y posibilitándoles un espacio de actividades terapéuticas que acompañan el desarrollo del tratamiento prescripto.

Por otra parte y siguiendo con el análisis de los factores que dieron origen a la creación de los talleres, los coordinadores se refieren al impacto de la crisis social y económica que atravesó la Argentina a finales de la década del 90 (Belmartino, 2005) y a la aparición de un nuevo tipo de paciente:

Lo que nosotros empezamos a ver era que nos llegaba una población al hospital absolutamente inédita, que era gente, sobretodo hombres, que habian empezado a perder sus puestos de trabajo, que por ahi los tenían hace 20, 30 años y se quedaban, a los 40, 50 años sin su puesto, sin obra social, con estados depresivos reactivos a esa situación de desempleo, cuando nunca tenían antecedentes de esa patología, y que aparecian un montón de situaciones orgánicas, en paralelo a enfermedades orgánicas simultánea a esta situación ... y la verdad es que no dábamos abasto, empezábamos a sentir que no teníamos recursos. (Julieta, coordinadora)

Las derivaciones a talleres alternativos les permitieron satisfacer un tipo de demanda que a partir de la década del 90 fue inédita y creciente, en cuya atención directa no se ven involucrados los profesionales del área -que ya se encuentran saturados de trabajo- y que les permite dar respuesta a la instancia de atención de diversos tipos de pacientes. Es interesante mencionar, sin embargo, que la población a la que se refiere en este relato -varones mayores de 40 años, desempleados- no es significativa en cuanto a su participación actual en los talleres, a los que, como hemos señalado, asisten principalmente, mujeres mayores de 60 años.

\section{Participantes de los talleres}

a) Participación y asistencia

A partir de las entrevistas a los usuarios de los talleres, podemos señalar tres tipos de motivaciones 
referidas para explicar la participación en estas actividades, que presentamos organizadas en función de su repetición en los relatos: (a) búsquedas de bienestar general, (b) búsquedas de salud específica y (c) búsquedas espirituales.

El aspecto refiere una asistencia a los talleres en búsquedas de bienestar, asociados a los efectos positivos de la participación en redes sociales y a la creación de vínculos con pares. La asistencia estable a los grupos brinda la pertenencia a un grupo social y promueve, desde la perspectiva de los usuarios, conductas saludables. Este último hecho es considerado terapéutico en sí mismo, como puede observarse en el relato de Ofelia, una mujer de 72 años, jubilada y viuda desde hace dos años:

A mi me hace mucho bien ir al taller de Yoga. Yo voy porque participar en el grupo me obliga a salir de casa, a tener un horario, una actividad, mis tiempos propios... a escapar de mis hijos que me dejan mis nietos para que los cuide todo el día.... a tener mis propias actividades, un tiempo.... y un lugar que son sólo para mí. (Ofelia, usuaria del taller de Yoga)

$\mathrm{O}$ en el caso de Oscar - 72 años, viudo y jubilado-, quien nos decía:

Aunque parezca mentira, ir al taller me obliga a cuidarme más, a afeitarme, a tener ropa limpia...a ponerme presentable para ir. Eso me hace bien, porque desde que enviudé tiendo a quedarme en casa y estar solo y eso no me hace bien. Porque cuando enviudé me había dado por quedarme encerrado, pasaba mucho tiempo sentado en la cocina, mirando el reloj y viendo la vida pasar. (Oscar, usuario de talleres de Tango, Vínculos y Danzas Circulares)

En ambos relatos se traduce el valor asignado a los talleres como un medio para obtener un ámbito y un tiempo propios, que les permiten a los sujetos la realización de una actividad.

El segundo aspecto asocia su asistencia a búsquedas de salud para hacer frente al tratamiento de un amplio espectro de dolencias, referidos como enfermedades crónicas, enfermedades propias de la vejez, entre otras. Teniendo en cuenta que este segundo grupo con el que trabajamos ya ha sido diagnosticado de una dolencia y por lo tanto asiste al hospital para su tratamiento, se atribuye la eficacia de la complementariedad a la combinación de ambos tipos de prácticas terapéuticas.

Es interesante destacar que, si bien los talleres surgen en el área de Salud Mental, especialistas de otras ramas de la biomedicina recomiendan a sus pacientes la utilización de los talleres abiertos a la comunidad, en virtud de considerarlos complementarios de los tratamientos que ellos efectúan.

Empecé a ir porque la vecina, la señora de acá al lado, yo la veía mucho mejor, como más vital, de mejor humor, y un día le pregunté que estaba haciendo...ella me contó que en el hospital iba a las clases de Tai Chi Chuan. Yo al principio no sabía ni qué era, pero como la veía tan bien a ella y me invitó, me animé. Ella tenía mucho dolor de espalda y tomaba muchos remedios. $Y$ me dijo que estaba tomando menos remedios eso me entusiasmó. (Ester, usuaria del taller de Tai Chi Chuan)

La combinación de remedios alopáticos y prácticas alternativas pareciera actuar de modo eficaz en diferentes niveles de la enfermedad, por lo que no se comprende como equívoco o incoherente la sumatoria de estrategias terapéuticas. En tal sentido, como notara Maluf (2005), los usuarios de las terapias alternativas poseen una visión de la salud que involucra aspectos físicos y espirituales, a esto es a lo que se le denomina "mirada holística". Por el contrario, el mismo término en biomedicina suele atribuirse al hecho de comprender al hombre en la interrelación de todos sus sistemas biológicos, acotando la idea al límite de lo corporal tangible (Douglas, 1998).

Finalmente, en cuanto al tercer aspecto, si bien los usuarios que asisten a los talleres hospitalarios por búsquedas de carácter espiritual representan una minoría, no por ello deja de ser interesante analizar su caso, en virtud de que manifiesta un fenómeno de resignificación de las lógicas y marcos de ambos tipos de medicinas, tanto de la biomedicina como de las terapias alternativas.

Creo que la meditación que se hace en el Yoga te lleva a otros estados de conciencia...estados más tranquilos en los que podes escucharte y encontrar la raiz de tus problemas emocionales, que luego se hacen carne y enfermedad en el cuerpo...también es el camino para deshacer el karma, para evolucionar y prepararte espiritualmente. En el hospital hacer Yoga es gratis, y yo no puedo pagar un taller afuera. (Analía, usuaria del taller de Yoga)

De acuerdo con lo explicitado por los usuarios, los motivos de selección y asistencia a los talleres alternativos se centran en la posibilidad de acceder a una terapia alternativa específica sin costo económico alguno. Asimismo, en algunas ocasiones se menciona en un sentido positivo- la certeza de que el especialista posee una buena formación en su disciplina y ha sido, además, avalado por la institución hospitalaria para ejercer su actividad. Las terapias alternativas seleccionadas por estos participantes son Yoga, Tai Chi Chuan, Bioenergética, Meditación con Cuencos 
Tibetanos y Meditación Trascendental. En estos casos no estamos frente a estrategias de complementariedad terapéutica en un sentido estricto, porque en ellos el uso de la disciplina alternativa se asocia con búsquedas individuales relacionadas al campo espiritual y no con la selección de prácticas para tratar la enfermedad.

Siguiendo con esta línea de análisis, nos parece interesante señalar que, en esta última tipología no se observa-al menos desde la perspectiva de los usuariosel proceso de domesticación que, como notara Fadlon (2005) sufren ciertas terapias alternativas al inscribirse en contextos biomédicos; proceso que implica una apropiación de saberes y prácticas que, siendo completamente ajenas al campo biomédico, son tomadas por él. En tal sentido, los aspectos rituales y los marcos filosóficos de las disciplinas alternativas que suelen enmascararse, licuarse y desaparecer como resultado del contacto institucional con la biomedicina (Mc Guire \& Kantor, 1988) permanecen presentes en la percepción de estos usuarios, quienes estrictamente refieren sus motivos de elección y asistencia a lo que se ha denominado los aspectos más "esotéricos" y "espirituales" de las terapias alternativas.

Este es un hecho interesante a destacar ya que, pese al pedido expreso de los coordinadores de que en el dictado de los talleres se mantenga un lenguaje simple y desapegado, que obvie menciones a contenidos espirituales y energéticos que remitan a otras ideas de salud y enfermedad, este último grupo de usuarios destaca justamente estos contenidos como parte de lo aprendido en el taller.

\section{b) Definiciones sobre la salud y la enfermedad}

En los relatos de los usuarios, es difícil rescatar definiciones claras sobre la salud y la enfermedad. Al ser interrogados por estas cuestiones, los entrevistados refirieron asociaciones directas a las condiciones y características del grupo etáreo al que pertenecen. Recordemos que la mayoría de los asistentes son mayores de 60 años y que se encuentran jubilados. En este sentido, la idea de salud remite la mayor parte de las veces a la idea de mantenerse activo en función de la edad. La posibilidad de participar en un grupo, realizar una práctica saludable, realizar actividad física suave contribuye a una idea de bienestar frente a las dolencias-asociadas a la edad- a la aceptación de las pérdidas y a la posibilidad de generar nuevos vínculos.

Para mí, estar sano es sentirme lo mejor posible con lo que me toca por la edad que tengo...y ya no soy un jovencito y hay dolores y molestias que son lógicas para mi edad. Para mí, estar como a los 40 es imposible, pero trato de estar lo mejor posible. (Oscar usuario de los talleres de Tango, Vínculos y Danzas Circulares)
Para mí, juntarme con un grupo de gente a hablar es saludable, no estar tan solo, que como me dice el médico eso me hace mucho mal. Yo creo que estar solo en mi casa y no tener nada que hacer es lo que me enferma y antes no me daba cuenta. (José, usuario del taller de Tai Chi Chuan)

A partir de los relatos recogidos en el trabajo de campo, podemos conceptualizar dos niveles de atribución de la enfermedad: un primer nivel se asocia a lo inevitable, el deterioro y las enfermedades propias de la edad; un segundo nivel refiere a la enfermedad como resultante de la falta de actividad y el aislamiento que en la mayoría de los casos, es correlato de la pérdida de actividades laborales y familiares. Nos interesa resaltar que entre los usuarios de los dos primeros grupos -aquellos que asisten en búsqueda de bienestar y aquellos que lo hacen por cuestiones específicas de salud-, hay una atribución de la idea de salud y enfermedad mayoritariamente biomédica. En sus relatos, los términos que definen las características de las situaciones saludables y de la dolencia, traducen a un lenguaje cotidiano la terminología biomédica. En ese contexto, aparecen menciones a nociones del campo alternativo, como armonía, energía, chakras, aura, y se asocian al bienestar de la práctica desde un lenguaje llano y atravesado por el campo biomédico en donde esta se realiza.

c) La experiencia de combinar biomedicina y alternativas

Como cierre de las entrevistas, les pedimos a los usuarios que nos comentaran acerca de la experiencia personal asociada a los talleres y la reflexión de la complementariedad entre lo biomédico y los talleres alternativos.

Respecto de la experiencia de participación en los talleres, las respuestas marcan una correlación directa con los motivos de asistencia y las nociones de salud y enfermedad. En tal sentido, la continuidad en el taller se asocia a que la práctica elegida/recomendada genera bienestar, término que se asocia a una mirada holista de la atención de la salud. La complementariedad terapéutica entre talleres alternativos y biomedicina no aparece -desde la perspectiva de los usuarios- como un ámbito de tensiones o conflicto; las distinciones entre ambos campos terapéuticos no son relevantes, es la experiencia de bienestar la que da lugar a estas prácticas de complementariedad.

Al momento de reflexionar sobre el fenómeno no aparece la tensión de los campos terapéuticos, sino que aparece la idea de bienestar asociada a la combinación. Así es que a criterio de los usuarios de los talleres alternativos, las medicinas combinadas actuarían en 
distintos niveles de la enfermedad, proporcionando la experiencia de holismo en el tratamiento, al posibilitar la atención de distintas esferas de la dolencia.

Este hecho permite pensar que los usuarios son ajenos a la discusión implícita entre coordinadores y especialistas alternativos acerca de la legitimidad de uno u otro modelo terapéutico. Como usuarios, presentan una mirada pragmática, aprovechando las ofertas disponibles.

\section{Conclusiones}

Desde distintos ámbitos se plantea y reafirma que aquello que se denomina "bienestar" no se circunscribe únicamente la ausencia de malestar, sino que se trata de un término que evoca una amplitud tal de factores y que, por tal motivo, es difícil de mensurar o estandarizar. Es así que la relación malestar-terapéutica supera los términos meramente biológicos, y se habilita a incluir modos cada vez más heterogéneos de búsquedas del bienestar.

En el contexto de los hospitales públicos de la Ciudad de Buenos Aires las actividades permitidas se encuentran reguladas bajo la ley del ejercicio de las actividades médicas y disciplinas afines, limitando el diagnóstico y tratamiento de las dolencias a profesionales médicos o a las llamadas "disciplinas auxiliares" de la biomedicina. Sin embargo, a partir de nuestra experiencia de campo pudimos dar cuenta de que estas limitaciones legales no excluyen totalmente la posibilidad de inserción de otro tipo de abordajes en el contexto hospitalario, lo cual se evidencia, de manera concreta, a partir de la promoción por parte del gobierno local de talleres alternativos. A partir de diferentes entrevistas realizadas en este contexto pudimos notar, asimismo, que, más allá de las diferencias en relación a su significado, la idea de bienestar aparecía de manera recurrente en los relatos de usuarios, talleristas y coordinadores. De este modo, la búsqueda de bienestar aparece como un criterio importante en los motivos de elección, derivación y consumo de las medicinas alternativas/ complementarias en el ámbito hospitalario.

Pese a que el porcentaje numérico de las personas que asisten a los talleres no es significativo en relación con la población total de los hospitales, la aparición de las terapias alternativas en el ámbito hospitalario es un hecho que consideramos novedoso. Es así que en el ámbito de talleres alternativos en hospitales, conviven actualmente miradas muy distintas sobre fenómenos aparentemente concretos, como el de la enfermedad y la salud. Pudimos rescatar, a partir del relato de los entrevistados -coordinadores, especialistas alternativos y usuarios-, que existen importantes diferencias de atribución y sentido de las diversas nociones que hacen a la comprensión del proceso de enfermedad y terapia. Sin embargo, pese a que las contradicciones en estas cuestiones que parecieran ser centrales, encontramos que es posible cohesionar estas miradas a partir de perspectiva del usuario y de la amplitud del concepto de bienestar, ya que atraviesa las distancias de sentido y la lejanía de conceptos teóricos, aunando mediante la experiencia de la búsqueda de bienestar y salud, culturas terapéuticas disímiles y hasta contradictorias. En la experiencia del usuario, las diferentes disciplinas englobadas en la búsqueda de bienestar dejan de lado cuestiones teóricas y técnicas, permitiendo la integración y la posibilidad de ingresar, así al campo de la complementariedad.

\section{Referencias}

Ai, A. L. \&. Bolling, S. F. (2002). The use of complementary and alternative therapies among middle-aged and older cardiac patients. American Journal of Medical Quality, 17(1), 21-27.

Albanese, C. (1990). Nature religion in America: From the Algonkian Indians to the New Age. Chicago, IL: The University of Chicago Press.

Alexander, K. (1992). Roots of the New Age. In J. Lewis \& J. Melton (Eds.), Perspectives in the New Age (pp. 30-47). Albany: State University of New York Press.

Belmartino, S. (2005). La atención médica argentina en el Siglo $X X$. Instituciones y procesos. Buenos Aires: Siglo XXI.

Berenzon Gorn., S., Navarro, S. A., \& Saavedra Solano, N. (2009). El uso de las terapias alternativas y complementarias en población mexicana con trastornos depresivos y de ansiedad: resultados de una encuesta en la Ciudad de México. Salud Mental, 31(2),107-115.

Boon, H., Brown, J., Gavin, A., Kennard, M., \& Stewart, M. (1999). Breast cancer survivors' perceptions of complementary/alternative medicine (CAM): Making the decision to use or not to use. Qualitative Health Research, 9, 639-653.

Broom, A., \& Tovey, P. (2008). Therapeutic pluralism: Exploring the experiences of cancer patients and professionals. London: Routledge

Carozzi, M. J. (2001). Nueva Era y terapias alternativas. Construyendo significados en el discurso y la interacción. Buenos Aires: Ediciones de la Universidad Católica Argentina.

Cartwright, T. \& Torr, R. (2005). Making sense of illness: The experiences of users of complementary medicine. Journal of Health Psychology, 10, 559-572

Douglas, M. (1998). Estilos de pensar. Barcelona: Gedisa.

Eisenberg, D., Davis, R., Ettner, S., Appel, S., Wilkey, S., Van Rompay, M., \& Kessler, R. (1998). Trends in alternative Medicine Use in the United States, 1990-1997. JAMA, 280(18),1569-1575.

Fadlon, J. (2005). Negotiating the holistic turn: The domestication of alternative medicine. Albany: State University of New York. 
Freidin, B. \& Timmermans, S. (2008). Complementary and alternative medicine for children's asthma: Satisfaction, care provider responsiveness, and networks of care. Qualitative Health Research, 18, 43-55.

Furnham, A. \& Smith, C. (1988). Choosing alternative medicine: A comparison of the relieves of patients visiting general practitioner and a homeopath. Social Science \& Medicine, 26, 685-689.

Heelas, P. (1996). The New Age movement: The celebration of the self and the sacralization of modernity. Oxford, UK: Blackwell Publishers.

Idoyaga Molina, A. (1997). Ethnomedicine and world-view. A comparative analysis of the rejection and incorporation of the contraceptive methods among Argentine women. Anthropology \& Medicine, 4, 145-158.

Idoyaga Molina, A. (2008). Las medicinas tradicionales en el Noroeste Argentino. Reflexiones sobre síntesis de praxis y saberes biomédicos, rituales católicos y fragmentos de creencias indígenas. Ketzalkalli, 1, 69-92.

Illouz, E. (2010). La salvación del alma moderna. Terapia, emociones y la cultura de la autoayuda. Madrid: Editorial Katz.

Jorm, A. F. (2000). Mental health literacy: Public knowledge and beliefs about mental disorders. British Journal of Psychiatry, 177, 396-401.

Karasz, A. \& Dempsey, K. (2008). Health seeking for ambiguous symptoms in two cultural groups: A comparative study. Transcultural Psychiatry, 45(3), 415-438.

Korman, G. P. \& Idoyaga Molina,A. (2010). Cultura y Depresión. Aportes antropológicos para la conceptualización de los trastornos mentales. Buenos Aires: Akadia.

Korman, G. P. \& Saizar, M. (2006). Reflexiones en torno a la inclusion del yoga como terapia complementaria en los tratamientos psicoterapéuticos cognitivos en Buenos Aires. Revista Brasileira de Sociologia da Emoção, 5(13), 97-110.

Maluf, S. W. (2005). Mitos coletivos, narrativas pessoais: Cura ritual, trabalho terapêutico e emergência do sujeito nas culturas da "Nova Era". Mana, 11(2), 499-528.

Mamtani, R. \& Cimino, A. (2002). A primer of complementary and alternative medicine and its relevance in the treatment of mental health problems. Psychiatric Quarterly, 73, 367-381.

Martins, P. (1999). As terapias alternativas e a libertação dos corpos. In M. J. Carozzi (Ed.), A nova era do Mercosul (pp. 80-105). Petrópolis, RJ: Vozes.

Mc Guire, M. \& Kantor, D. (1988). Ritual healing in Suburban America. New Brunswick, NJ: Rutgers University Press.

Montgomery, K. (2006). How doctors think: Clinical judgment and the practice of medicine. Oxford, UK: Oxford University Press.

National Institute of Health. (2003). What is complementary and alternative medicine? National Center for Complementary and Alternative Medicines. Acceso en 5 de diciembre, 2012, en http://nccam.nih.gov/sites/nccam.nih.gov/files/ informaciongeneral.pdf

O'Connor, B. (1995). Healing traditions: Alternative medicine and the health professions. Philadelphia: University of Pennsylvania Press.

Organización Mundial de la Salud (2002). Traditional medicine strategy 2002-2005. Ginebra, Suiza: Author.

Saizar, M. (2009). De Krishna a Chopra. Filosofias y prácticas del Yoga en Buenos Aires. Buenos Aires: Editorial Antropofagia.
Saizar, M., Bordes, M., \& Sarundiansky, M. (2010). El cuidado en los márgenes. Nuevas formas de voluntariado en el contexto del Estado Post-Social. In B. Lorente (Ed.), Transformaciones del Estado Social. Perspectivas sobre la intervención social en Iberoamérica (pp. 199-254). Buenos Aires: Editorial Miño y Davila.

Sharma, C. (2000). Medical pluralism and the future of CAM. In M. Kelner, B. Wellman, B. Pescosolido \& M. Saks (Eds.), Complementary and alternative health care: challenge and change (pp. 211-222). Amsterdam, Netherlands: Harwood.

Shimazono, S. (1999). New Age movement or new spirituality movements and culture. Social Compass, 46, 121-133.

Siahpush, M. (1998). Postmodern values, dissatisfaction with conventional medicine and popularity of alternative therapies. Journal of Sociology, 34, 58-70.

Thorne, S. (2000). Data analysis in qualitative research. Evidence Based Nursing, 3, 68-70.

Vincent, C. \& Furnhman, A. (1996). Why do patients turn to complementary medicine? An empirical study. British Journal of Clinical Psychology, 35, 37-48.

Zollman, S. \& Vickers, A. (1999) ABC of complementary medicine. What is complementary medicine?, $B M J, 319$, 693-696.

Recebido em: 18/03/2013

Aceite em: 17/06/2013

María Mercedes Saizar es Doctora en Cultura y Sociedad (CAEA/IUNA). Investigadora en el Consejo Nacional de Investigaciones Científicas y Técnicas - CONICET, y miembro del Centro Argentino de Etnología Americana - CAEA. Dirección: Av. Rivadavia 1917 (C1033AAJ). Ciudad Autónoma de Buenos Aires. República Argentina. Email: m_saizar@yahoo.com

Mercedes Sarudiansky es Licenciada y Doctora en Psicología (Universidad de Buenos Aires/Universidad Nacional de San Luis, Argentina). Docente en la Universidad de Buenos Aires - Facultad de Psicología. Email: msarudi@gmail.com

Guido P. Korman es Licenciado y Doctor en Psicología. Investigador en el Consejo Nacional de Investigaciones Científicas y Técnicas - CONICET, y miembro del Centro Argentino de Etnología Americana - CAEA, (Argentina). Docente de grado y posgrado - Universidad de Buenos Aires - Facultad de Psicología. Email: gkorman@conicet.gov.ar

\section{Como citar:}

Saizar, M. M., Sarudiansky, M., \& Korman, G. P. (2013). Salud mental y nuevas complementariedades terapéuticas. La experiencia en dos hospitales públicos de Buenos Aires, Argentina. Psicologia \& Sociedade, 25(2), 451-460. 